\title{
Novel and conventional assays in determining abundance of Streptococcus mutans in saliva
}

\begin{abstract}
Objective: This study aimed to comparatively evaluate the validity of a novel immunoassay and a conventional assay in detecting salivary Streptoccous mutans (S. mutans) in a pediatric cohort.
\end{abstract}

Methods: A total of 190 children aged 3-4 years were recruited from three kindergartens representing low, moderate, and high socio-economic groups in Hong Kong. The abundance of S. mutans in the saliva samples was analyzed with three assay systems viz. a conventional culture-based assay (Dentocult SM), a novel immunoassay system (Saliva-Check mutans) based on monoclonal antibody technology, and a Taqman real-time PCR assay taken as a gold standard.

Results: The immunoassay accurately categorized salivary samples into two groups with high $\left(\geqq 5 \times 10^{5} \mathrm{CFU} / \mathrm{ml}\right)$ and low $\left(<5 \times 10^{5} \mathrm{CFU} / \mathrm{ml}\right)$ S. mutans levels. The sensitivity/ specificity was $97.6 \% / 90.6 \%$. The conventional culture-based assay reached a reasonably high sensitivity/specificity $(92.8 \% / 81.3 \%)$ in identifying children with moderate $\left(\geqq 10^{4} \mathrm{CFU} / \mathrm{ml}\right) \mathrm{S}$. mutans level. However, its sensitivity/specificity in selecting children with high $\left(\geqq 10^{5}\right.$ $\mathrm{CFU} / \mathrm{ml})$ and very high $\left(>10^{6} \mathrm{CFU} / \mathrm{ml}\right)$ S. mutans levels were not sufficient $(78.7 \% / 79.8 \%$ and $25.8 \% / 91.8 \%$, respectively).

Conclusions: The monoclonal antibody-based immunoassay, accurately and rapidly determining $S$. mutans abundance in saliva, could be useful for assessing caries risk of patients. 


\section{Introduction}

The use of salivary biomarkers for identifying caries-susceptible individuals is of great interest to dental professionals, while they are striving toward cost-effective caries control and optimized dental treatment planning. ${ }^{1}$ Since the central role of Streptococcus mutans (S. mutans) in the initiation of caries has been well established ${ }^{2,3}$, abundance of $S$. mutans in saliva is one of the most commonly used microbiological parameters for assessing caries risk.

A variety of test assays are commercially available for measuring the abundance of $S$. mutans in saliva. ${ }^{4,5}$ Of these widely used are the culture-based detection assays. A noted drawback of these culture-based methods is that they are not sufficiently specific to $S$. mutans. Culture-based methods detect not only $S$. mutans, but also other members of the mutans Streptococcus group and even some non-Streptococcus species, most of which are not cariogenic. ${ }^{4,5}$ Moreover, conventional culture-based assays normally require 2-4 days to obtain a result as cultures need to be incubated for sufficient time before enumeration. ${ }^{4}$ This appears to be inconvenient for patients, clinicians and public health workers, hampering the wide applications of these products.

Recently, a $S$. mutans detection system Saliva-Check ${ }^{\circledR}$ mutans immunoassay (GC, Japan) has been introduced. This assay is based on monoclonal antibodies specific for $S$. mutans and the detection depends on antigen-antibody reactions. ${ }^{6}$ Hence, the immunoassay has been designed in such as way that it can offer highly specific and immediate outcome (in 15 minutes). Thus, it appears to be a promising tool for caries risk assessment in the community and clinical settings.

The original Saliva-Check ${ }^{\circledR}$ mutans system was designed to categorize S. mutans abundance into three levels (high, moderate, and low), with the aid of a silver enhancer. A 
previous study in a small sample of young adults $(n=28)$ suggested a moderate correlation $(\mathrm{r}=0.63)$ between Saliva-Check ${ }^{\circledR}$ mutans readings and the $S$. mutans count, using "PCRconfirmed colony forming unit (CFU)" as a benchmark. ${ }^{7}$ A further comparison showed that the average $S$. mutans count (CFU/ml saliva) in samples classified as with "high" bacterial level was $3.4 \times 10^{6}+/-4.3 \times 10^{6}$, higher than those for the groups classified as "moderate" $(1.3 \mathrm{X}$ $\left.10^{5}+/-1.5 \times 10^{5}\right)$ and "low" (5.7 X $\left.10^{4}+/-1.1 \times 10^{5}\right)$ (both $\left.\mathrm{P}<0.05\right)$. Nevertheless, no significant difference was found between the latter two groups $(\mathrm{P}>0.05)$.

Based on these findings, the system has been refined into a two-category assessment outcome (i.e. "high" and "low" denoting $S$. mutans levels above and below $5 \times 10^{5} \mathrm{CFU} / \mathrm{ml}$ saliva, respectively). However, the validity of this modified immunoassay system has not been evaluated and compared to other detection method in a large-scale epidemiological study and thus is the objective of this study.

\section{Method and materials}

\subsection{Recruitment of participants}

An ethical approval for the study was granted by the University of Hong Kong Institutional Review Board (HKU/HA HKW IRB No: UW 08-400). After obtaining parents' written consents, 190 children aged 3-4 years were recruited from 3 kindergartens located in different districts representing low, moderate, and high socio-economic groups in Hong Kong.

\subsection{Determination of $S$. mutans abundance using three methods}

The abundance of $S$. mutans in children's saliva was determined by using a conventional culture-based assay Dentocult ${ }^{\circledR}$ SM strip (Orion Diagnostica, Finland), the immunoassay system Saliva-Check ${ }^{\circledR}$ mutans (GC, Japan), and the Taqman real-time PCR, which served as a 
gold standard. During the assessment with each of the three methods, the examiner was blinded to the results obtained from other two methods.

Refraining from foods, drinks, and toothbrushing for at least one hour, each child was instructed to chew paraffin pellet for one minute. The rough surface of the Dentocult ${ }^{\circledR} \mathrm{SM}$ strip was then pressed on his/her tongue dorsum for 10 times, before being removed between gently closed lips. The Dentocult ${ }^{\circledR}$ SM strip was transferred to the microbiological laboratory and incubated in a vial of selective culture broth under $37^{\circ} \mathrm{C}$ for 48 hours. The density of bacterial colony forming unit (CFU) was rated into four levels (Class 0-3) according to the standard reference chart provided by the manufacturer.

Saliva-Check ${ }^{\circledR}$ mutans immunoassay system was used to determine the abundance of $S$. mutans in saliva as a chair-side test on the spot. For this purpose, participants were requested to chew paraffin for 3 minutes after the saliva sampling with Dentocult ${ }^{\circledR}$ SM strips. Stimulated saliva was collected. A $250-\mu 1$ saliva was treated with $50 \mu 1$ of Tris- $\mathrm{NaOH}$ (Reagent-1) for 30 seconds vigorously, and then mixed with $100 \mu 1$ of Tris-citrate (Reagent-2) to neutralize the $\mathrm{pH}$. A $100 \mu 1$ sample of the treated saliva was applied to the test device. A red line appearing on the test device after 15 minutes indicated a high $S$. mutans level. If no line was visible, the $S$. mutans level was classified as low.

The remaining portion of the stimulated saliva was transferred to the laboratory and the S. mutans level was determined by quantitative species-specific $5^{\prime}$ fluorogenic exonuclease (Taqman) real-time PCR (Q-RT-PCR). DNA was extracted from salivary samples as previously described. ${ }^{8}$ DNA extracted from $S$. mutans ATCC cultures (ATCC 35668 and ATCC 700610) was used as the positive control. Q-RT-PCR was performed in ABI PRISM 7900HT sequence detection system using species-specific Taqman probes (Applied Biosciences). Taqman assay 
was further validated to quantify the $S$. mutans counts in the range of $10^{3}$ to $10^{9}$ cells. Each PCR was performed in a total volume of $20 \mu$ consisting $10 \mu 1$ of Taqman Gene expression master mix, $1 \mu 1$ of forward and reverse primers, $1 \mu 1$ of Taqman probe, $1 \mu 1$ of template DNA, and appropriate amount of sterilized DNase-RNase-free water. The cycling conditions were 50 ${ }^{\circ} \mathrm{C}$ for 2 minutes, $95^{\circ} \mathrm{C}$ for 10 minutes and 40 cycles at $95{ }^{\circ} \mathrm{C}$ for 15 seconds, $60^{\circ} \mathrm{C}$ for 1 minute.

\subsection{Data analysis}

The data were analyzed with the Statistical Package for Social Sciences (SPSS, version 17.0). Comparison was made between the mean $S$. mutans counts of two groups defined by Saliva-Check ${ }^{\circledR}$ mutans as with low and high bacterial load. Non-parametric method (MannWhitney test) was applied since the assumption of homogeneity of variance was violated, as shown by the Levene test. For Dentocult ${ }^{\mathbb{R}}$ SM, means of the four categories (Class 0-3) were compared by using parametric test (post hoc Tukey test), since both the normal distribution and homogeneity of variance were supported. The validity of the immunoassay system and conventional culture-based assay in determining $S$. mutans abundance was evaluated and compared. The main parameters used included sensitivity, specificity, and Youden's index (sensitivity + specificity -1 ), which is a single statistic that captures the overall performance of a diagnostic test. ${ }^{9}$ A Youden's index above 0.6 or above 0.8 indicates a "useful" or "good" test, respectively ${ }^{10,11}$.

\section{Results}

Table 1 shows the mean number of $S$. mutans $[\log 10(\mathrm{CFU} / \mathrm{ml}$ saliva $)]$ confirmed by realtime PCR in each classification of both test assays (Saliva-Check ${ }^{\circledR}$ mutans and Dentocult ${ }^{\circledR} \mathrm{SM}$ strip). The immunoassay system Saliva-Check ${ }^{\circledR}$ mutans indicated 136 (71.6\%) and 54 (28.4\%) children harbored low and high levels of $S$. mutans, respectively. The mean $S$. mutans count $[\log 10(\mathrm{CFU} / \mathrm{ml})]$ was $3.44+/-0.84$ for the former group, significantly lower than that of the 
latter group, which was $5.97+/-0.97(\mathrm{P}<0.001)$.

The conventional assay Dentocult ${ }^{\circledR}$ SM strip classified $S$. mutans counts into four groups in an ascending order from $<10^{4} \mathrm{CFU} / \mathrm{ml}$ to $>10^{6} \mathrm{CFU} / \mathrm{ml}$, ' 0 ' being the lowest and ' 3 ' being the highest. When Taqman real-time PCR assay was used as the gold standard, the S. mutans levels $[\log 10(\mathrm{CFU} / \mathrm{ml})]$ of these four categories appeared to be $3.10+/-0.56$ (Class 0$), 4.91+/$ 1.67 (Class 1), $5.20+/-1.19$ (Class 2), and $5.38+/-1.07$ (Class 3), respectively. While children in "Class 0" had significantly lower number of S. mutans, compared with the rest of children $(\mathrm{P}<0.001)$, there was no significant difference among Class 1-3 $(\mathrm{P}>0.05)$.

Saliva-Check ${ }^{\circledR}$ mutans immunoassay accurately categorized the saliva samples into two groups with high $\left(\geqq 5 \times 10^{5} \mathrm{CFU} / \mathrm{ml}\right)$ and low $\left(<5 \times 10^{5} \mathrm{CFU} / \mathrm{ml}\right) S$. mutans levels (Table 2). The sensitivity/specificity was $97.6 \% / 90.6 \%$. The Youden's Index was 0.882 . Dentocult ${ }^{\circledR}$ SM strip reached reasonably high sensitivity/specificity $(92.8 \% / 81.3 \%)$ and Youden's Index $(0.741)$ in selecting children with moderate $\left(\geqq 10^{4} \mathrm{CFU} / \mathrm{ml}\right)$ S. mutans level. However, it did not sufficiently identify children with high $\left(\geqq 10^{5} \mathrm{CFU} / \mathrm{ml}\right)$ and very high $\left(>10^{6} \mathrm{CFU} / \mathrm{ml}\right) S$. mutans levels. The sensitivity/specificity were $78.7 \% / 79.8 \%$ and $25.8 \% / 91.8 \%$, respectively. Both Youden's indexes were below 0.6. The contingency table (Table 3) compares the classifications by the immunoassay $\left(10^{5} \mathrm{CFU} / \mathrm{ml}\right.$ as the cut-off point) and culture-based assay (under the best cut-off point of $\geqq 10^{4} \mathrm{CFU} / \mathrm{ml}$ ) against the real-time PCR results.

\section{Discussion}

Although S. mutans is an important parameter for assessing caries risk, its clinical application is limited partly due to the lack of a highly accurate test which can be performed with minimal training and labor. This study, utilizing a relatively large sample and the Taqman real-time PCR technology as the gold standard, comparatively evaluated the validity of a 
promising novel immunoassay and a conventional assay. Taqman real-time PCR assay, as an advanced molecular detection technology, is considered one of the best techniques to quantify the bacterial load in clinical samples. ${ }^{12}$

Our results revealed a high validity of the Saliva-Check ${ }^{\circledR}$ mutans immunoassay system in determining $S$. mutans abundance in saliva. In the present cohort, the system accurately identified about one quarter $(28 \%)$ of children harboring high level ( $\geqq 5 \times 10^{5} \mathrm{CFU} / \mathrm{ml}$ saliva) of $S$. mutans. The sensitivity and specificity were both above $90 \%$. The Youden's index was 0.882. In light of the current polarized distribution of caries, selecting one quarter of the population with high risk for targeted prevention and intervention is a rational approach for achieving cost-effective caries control and optimized treatment planning. ${ }^{13}$

Comparatively, Dentocult ${ }^{\circledR}$ SM strip, with a four-class reading, is meant to generate a more refined, semi-quantitative assessment of bacterial abundance. However, our results indicated a lack of difference in $S$. mutans level among Class 1-3, despite the difference between Class 0 and the rest. Correspondingly, Dentocult ${ }^{\circledR}$ SM strip reached a reasonably high sensitivity/specificity (92.8\%/81.4\%) and Youden's index (0.741) only in identifying children with $S$. mutans level of $10^{4} \mathrm{CFU} / \mathrm{ml}$ and above (Class 1-3). Our findings suggest that a binary rating system (i.e. $\geqq 10^{4} \mathrm{CFU} / \mathrm{ml}$ or $<10^{4} \mathrm{CFU} / \mathrm{ml}$ ) of Dentocult ${ }^{\circledR} \mathrm{SM}$ strip may better reflect the abundance of $S$. mutans, the main cariogenic bacterial species.

The emergence of molecular detection methods has been one of the landmark revaluations in science over the past century. ${ }^{14}$ In the medical arena, these methods offer superior specificity and sensitivity over the conventional culture-based techniques in enumerating microorganism and molecules of health relevance. However, laboratory molecular methods, such as Taqman real-time PCR, are expensive and require specialized skills and equipments. The development 
of the immunoassay system for detecting $S$. mutans is in fact an example of introducing the molecular detection method into practical applications at chairside. The immunoassay system is characterized by its rapidity and ease of the testing procedures, which can be completed by a dentist or dental auxiliary. Since no cultivation is needed, the test requires no additional apparatus and minimizes the chance of contaminations. The immunoassay assays can be easily stored under room temperature for at least two years, while the conventional culture-based assays require special storage condition $\left(2-8^{\circ} \mathrm{C}\right.$; protected from light etc), with a shelf life normally no more than 6 months.

Although the immunoassay system provides a practical solution for accurately measuring S. mutans, the primary pathogen for caries, it should be borne in mind that $S$. mutans is not the only cariogenic bacterial species related to caries. ${ }^{15}$ For further development of the system, immunoassays for detecting other cariogenic species (e.g. Streptococcus sobrinus) may be considered. In addition, abundance of cariogenic bacteria should be synthesized with other parameters (diet, fluoride etc) for a better understanding of individual's caries risk.

\section{Conclusions}

The monoclonal antibody-based immunoassay, accurately and rapidly determining $S$. mutans abundance in saliva, could be useful for assessing caries risk of patients.

\section{Acknowledgements}

The immunoassay products used in this study were donated by the GC Corporation. This study is financially supported by the University of Hong Kong (Small Project Fund; Ref No.10400751) and Hong Kong Food and Health Bureau (Health and Health Service Research Fund; Ref No. 07080741). 


\section{References}

1. Tanzer JM, Livingston J, Thompson AM. The microbiology of primary dental caries in humans. Journal of Dental Education 2001;65(10):1028-37.

2. Keyes PH. The infectious and transmissible nature of dental caries. Findings and implications. Archives of Oral Biology 1960;1:304-20.

3. Fitzgerald RJ, Fitzgerald DB. The microbiologic status of test animals in relation to caries research. In: Tanzer JM, editor, Animal models in cariology: proceedings of a Symposium and Workshop on Animal Models in Cariology. Washington DC: Information Retrieval Inc. 1981. p.89-95.

4. Tanabe Y, Park JH, Tinanoff N, Turng BF, Lilli H, Minah GE. Comparison of chairside microbiological screening systems and conventional selective media in children with and without visible dental caries. Pediatric Dentistry. 2006;28(4): 363-8.

5. Nishikawara F, Nomura Y, Imai S, Senda A, Hanada N. Evaluation of cariogenic bacteria. European Journal of Dentistry 2007;1(1):31-9.

6. Shi W, Jewett A, Hume WR. Rapid and quantitative detection of Streptococcus mutans with species-specifi c monoclonal antibodies. Hybridoma 1998;17:365-371.

7. Matsumoto Y, Sugihara N, Koseki M, Maki Y. A Rapid and Quantitative Detection System for Streptococcus mutans in Saliva Using Monoclonal Antibodies. Caries Research 2006;40:15-19.

8. Oho T, Yamashita Y, Shimazaki Y, Kushiyama M, Koga T. Simple and rapid detection of Streptococcus mutans and Streptococcus sobrinus in human saliva by polymerase chain reaction. Oral Microbiology and Immunology 2000;15(4):258-62.

9. Youden WJ. An index for rating diagnostic tests. Cancer 1950;3:32-35.

10. Schisterman EF, Perkins NJ, Liu A, Bondell H. Optimal cut-point and its corresponding Youden Index to discriminate individuals using pooled blood samples. Epidemiology 2005;16(1):73-81. 
11. Chu CH, Lo EC, You DS. Clinical diagnosis of fissure caries with conventional and laser-induced fluorescence techniques. Lasers in Medical Science 2010;25(3):355-62.

12. Suzuki N, Yoshida A, Nakano Y. Quantitative analysis of multi-species oral biofilms by TaqMan Real-Time PCR. Clinical Medical Research 2005;3(3):176-85.

13. Beck JD, Weintraub JA, Disney JA, Graves RC, Stamm JW, Kaste LM et al. University of North Carolina Caries Risk Assessment Study: comparisons of high risk prediction, any risk prediction, and any risk etiologic models. Community Dentistry and Oral Epidemiology 1992;20(6):313-21.

14. Elliott WH. Biochemistry and Molecular Biology. $4^{\text {th }}$ Edition. Oxford University. 2009.

15. Samaranayake LP. Oral microbiology. In. Essential Microbiology for Dentistry. $3^{\text {rd }}$ Edition. Elsevier. 2006. P. 253-306. 